\title{
SYNTHESIS OF CLASSIFICATION DISPERSION SPECTRA FROM STELLAR ATMOSPHERES THEORY AND FROM HIGH DISPERSION SPECTRA
}

\author{
A. W. IRWIN, C. T. BOLTON and R. F. GARRISON
}

David Dunlap Observatory, University of Toronto

\begin{abstract}
The ATLAS programme has been corrected and modified for use at low effective temperatures. A grid of unblanketed model atmospheres has been generated for the region G5-K5, V-II. A spectrum synthesis programme has been written for the calculation of flux vs wavelength for selected regions of the spectrum. Temperature distributions from the grid of continuum models and from published blanketed models will be used along with published oscillator strengths as input data for the spectral synthesis programme.

Spectrograms of MK standards have been taken at $12 \AA \mathrm{mm}^{-1}$ and $120 \AA \mathrm{mm}^{-1}$. The University of Toronto PDS microdensitometer system has been programmed to allow comparison between the synthesized spectra and the observed spectra to determine the effects of effective temperature, surface gravity, microturbulence, and abundance on classification criteria.
\end{abstract}

\section{DISCUSSION}

McCarthy: This is a most encouraging union of mathematical synthesis and observational spectroscopy.

What happens in the construction of the synthetic spectra for lines which lack determinations of oscillator strength. Is this line omitted or is some likely value assumed?

Garrison: We make the best guess we can. We don't expect the results to be unambiguous, but it is worthwhile to do the best we can and to try to interpret the results. We hope to learn something in the process.

Stephenson: For years I have been trying to learn if anyone knows the reason for the well-known depression of the continuum on the blue side of the G-band. I suspect this might be related to the violet opacity source in $C$ and $S$ stars, which sets in at the same position approximately. Does the fact that your synthetic spectra do not show the G-band break mean that its cause is indeed unknown, or that you have omitted something known?

Garrison: The synthetic spectra shown were actual $12 \AA \mathrm{mm}^{-1}$ spectrograms convolved with a $2 \AA$ slit to simulate $120 \AA \mathrm{mm}^{-1}$ spectra. We have yet to reduce the $120 \AA \mathrm{mm}^{-1}$ plates but the comparison between the $12 \AA \mathrm{mm}^{-1}$ reduced spectra, the synthesized atmospheres and the $120 \mathrm{~A} \mathrm{~mm}^{-1}$ spectra should show the same results. If they don't, we'll try to figure out why they don't.

Stephenson: Perhaps this means that it is difficult to use high-dispersion spectra to interpret low-dispersion ones.

Bell: The answer to Stephenson's question is, I think, that there are far fewer $\mathrm{CH}$ lines on the red side of the G-band compared to the blue side.

Houziaux: (1) What type of instrumental profile are you taking? It may depend pretty much on the instrument which has been used. For instance if it is a prismatic instrument you have to change the instrumental profile throughout the spectrum.

(2) What is general philosophy of such a work? Is it to determine the spectral characteristics of the MK types with the help of synthetic spectra? Why not compare directly synthetic spectra to observed spectra and forget about MK classification? There are far more parameters in synthetic spectra than in the spectral classification.

Garrison: (1) The spectrographs used are all grating instruments and the slit has been set so that 
the projected slit is slightly larger than the resolution of the plate. Irwin has not yet decided on a final profile to be used and it can be changed easily.

(2) We are comparing directly the synthesized classification dispersion spectra from high dispersion and from stellar atmospheres models with actual low dispersion spectra in order to understand some of the effects of changes in physical parameters (such as abundance, microturbulence) on classification dispersion spectra.

Bell: Why do you not try the instrumental profile $e^{-1 \Delta \lambda / q}$ advocated by $C$. Veth (the last BAN) instead of a Gaussian?

Secondly, I am worried that you can never see the continuum in the G-band region of your stars and this may mean that you can never convolve a $12 \AA \mathrm{mm}^{-1}$ spectrum to a $120 \AA \mathrm{mm}^{-1}$ one.

Garrison: Irwin is worrying about the profile and can easily change it, but has not yet decided which to use. I am sure he is aware of the work of Veth, but I will convey your comments to him.

The rectified tracings were obtained by fitting a continuum point at each end of the spectrum. We do not see a continuum point within the G-band even at $12 \AA \mathrm{mm}^{-1}$. 